\title{
Water transport through epoxy-based powder pipeline coatings
}

\author{
Hossein Zargarnezhad a, ${ }^{*}$, Edouard Asselin ${ }^{a}$, Dennis Wong ${ }^{b}$, C.N. Catherine Lam ${ }^{b}$ \\ a The University of British Columbia, Department of Materials Engineering, Department of Materials \\ Engineering, 309-6350 Stores Road, Vancouver, BC V6T 1Z4, Canada \\ ${ }^{b}$ Shawcor Ltd., 25 Bethridge Road, Toronto, ON, M9W 1M7, Canada \\ *Corresponding author: hossein.zargar@ubc.ca (H. Zargarnezhad)
}

\begin{abstract}
In this study, we examined the water permeation kinetics of two common epoxy-based powder coating systems for pipelines across a range of industrially-relevant temperatures (from room temperature to $\left.80^{\circ} \mathrm{C}\right)$. We exclusively analyzed the nonlinear dependency of water transport on the vapor concentration at $65^{\circ} \mathrm{C}$. The vapor transport analysis of epoxy coatings demonstrated a turning point around this temperature, perhaps due to self-association of water molecules. At higher temperatures, break-up of water clusters and plasticization of the polymer expedited the transport. We also examined microstructural changes of the epoxy network due to water transport and found evidence for irreversible damage to epoxy coatings under hydrothermal exposures. It appears that the combination of thermal exposure and internal stresses in the glassy epoxy leads to a phase separation of filler particles from the epoxy matrix, as well as to a distinctive cavity formation in the coating membrane. Our results indicate that hydrothermal exposure is likely to increase aggregate porosity of the coating and a conservative implementation of standard coating requirements is therefore reasonable to avoid early degradation issues. Analysis of wetstate permeation is not only crucial for protection of transport pipelines, but it also is of high relevance to process equipment and underground storage tanks.
\end{abstract}

\section{KEYWORDS}

Fusion bonded epoxy, Vapor transport, Epoxy network, Polymer hydration, Water clustering. 


\section{Introduction}

Water sorption and dynamic interactions between water and polymeric networks continue to attract scientific and technological interests to improve polymeric materials functionality and stability [1]-[4]. During water sorption and outgassing by polymeric coatings, the non-equilibrium glassy state of the polymer, which prompts a gradual relaxation in polymeric chains, allows an additional uptake of water over time [5]. The resultant relaxation inevitably affects the barrier properties of the glassy polymer, as well as its resistance to degradation [6], [7]. In coating technologies, consequences of water uptake may affect adhesion strength, which is a central parameter in mitigation of undesired corrosion issues caused by mass transport across the protective coating [8]. Despite being primary components for pipeline coatings due to their good adhesion performance, epoxy materials are susceptible to moisture-induced degradation in wet environments [9], [10]. To provide a near-ideal protection against hydration, hydrophobic polymer structures are commonly added to the coating system as exterior layers [11], [12]. Yet, we do not fully understand failures that are witnessed on either single or multi-layer coatings [13], [14].

Analysis of mass transport processes and their effects on degradation of pipeline coatings is scarce in the literature (see [15]) and studies linked with coatings' performances are usually focused on the disbonded areas upon local failures [16], [17]. In a recent extensive survey [18], we discussed the existing literature on wet-state diffusion systems and showed that mass transport rates of aggressive species surrounding an operating pipeline—such as gaseous and ionic permeants—may remarkably change due to polymer hydration. Fusion-bonded epoxy (FBE) is an example of a polymeric coating that is highly interactive with water. Some field evidence suggests that exposure to humid environments may lead to formation of defects such as microcracking and disbondment on the applied coating system [14]. However, no publication has yet been provided to actually investigate quantitatively the effect of microstructural transformation on mass transport after polymer hydration. Admittedly, FBE shows good adhesion and superior resistance to cathodic disbondment, even without protective measures such as cathodic protection [18]. But, it also has low impact resistance and a tendency to disbond from the steel substrate 
if exposed to water at high temperatures (e.g., $\left.65^{\circ} \mathrm{C}\right)$ [19]. High performance powder coating (HPPC) is an alternative technology that is relatively less sensitive to moisture-induced degradation compared to FBE. In HPPC, passive transport is slowed due to a uniform coverage of the glassy FBE by a compliant polyethylene (PE) topcoat [20]. However, gradual hydration of the FBE primer is anticipated in HPPC and the multi-layered coating might be subjected to degradation in long-term exposures to wet environments. This degradation is regardless of applied corrosion prevention methods (e.g., cathodic protection).

Water uptake and diffusion through epoxy coatings have been widely investigated in the literature, primarily to improve material properties during hygrothermal ageing [5], [21], [22]. The tendency of existing polar groups toward hydration can lead to nonlinearities in the vapor transmission-concentration relationship in hydrophilic membranes [23], [24]. A substantial increase in flux stems from a change in sorption process upon hygrothermal exposures; increasing hydration causes irreversible microdamage in the polymer network, which increase the amount of 'trapped' water in the pre-existing free volume (i.e., Langmuir type sorption) [5]. Loading polymers with additive fillers is a practical approach to enhance permeation performance of the resultant membrane by adjusting pore size and distribution [25], [26]. Layered silicate has been shown to reduce water absorption in epoxy resins, although the silicate itself is neutral to the diffusion coefficient of water [27]. Based on prior results regarding the effects of additives on vapor transport [28], we could suggest that there may not be a direct relationship between filler particles and water sorption modes such as clustering. Yet, it seems that hydrophilic fillers pull out from the polymeric matrix in wet conditions [29]. Such an effect has been observed on the surface microstructure of FBE films after seven months of ageing at $85^{\circ} \mathrm{C}$ [14]. Yet, little empirical evidence exists to relate crosssectional morphological changes to water permeation in epoxy coatings.

The objective of the following study was to characterize vapor transport through epoxy-based powder coating films. We carried out water vapor transmission (WVT) tests to directly assess water permeability through free coating films. We also analyzed water vapor permeability according to the activity of water (vapor pressure) for the critical temperature of $65^{\circ} \mathrm{C}$ to investigate permeation on the threshold of the 
coating plasticization. In addition, we investigated the extent of hydrothermal damage to the microstructure of the coating across a range of industrially-relevant test temperatures $\left(25-80^{\circ} \mathrm{C}\right)$. Since water is expected

to disrupt functional groups in the epoxy over time [19] and to affect microstructure of the coating throughout its transport, we also performed a qualitative analysis of the FBE microstructure after exposure to wet environments.

\section{Experiments and methods}

\subsection{Materials and characterization}

Various free-standing membranes with no intentional defects were investigated in this research. The water permeability of polymeric films was measured using a gravimetric cup method [30]. Free films of all the coating materials, including FBE, topcoat PE, and HPPC, were supplied by Shawcor Ltd. The FBE used in this study has a glass transition temperature $\left(T_{g}\right)$ of $110^{\circ} \mathrm{C}$ [31]. The coating membranes were cut individually into a circular plate, with a diameter of $7 \mathrm{~cm}$. Each sample was measured for thickness using the geometric test area with an ultrasonic thickness gauge (Phase-II PTG-3750) that has an accuracy of $2 \mu \mathrm{m}$, and results are presented as means \pm standard deviation.

For the analysis of water ingress into FBE, we followed an experimental procedure illustrated in Figure 1 and examined the coating microstructure of samples after each exposure condition. To investigate the effects of temperature and stress on the epoxy microstructure in wet environments, four free film samples and four FBE-coated steel panels (also supplied by Shawcor Ltd.) were immersed in a deionized (DI) water bath at $65^{\circ} \mathrm{C}$ ( $a$ and $b$, respectively, in Figure 1). Let us recall that this study is concerned with effects of water transport on the degradation of coatings (not external protective measures like cathodic protection). Thus, we did not investigate the performance of coated panels using cathodic protection throughout hydrothermal ageing. Free films (with $4 \mathrm{~cm}^{2}$-coated areas) and steel panel samples (with 15 $\mathrm{cm}^{2}$-coated areas) were aged for 30 and 90 days. Steel panels were then cross-sectioned using cutting fluids to prevent hydrolytic degradation during elevated temperature exposure and then prepared for microstructural study. Coating cross-sections before and after permeation and immersion tests were 
examined using a FEI Quanta 650 scanning electron microscope (SEM) in back-scattered electron (BSE) mode at an accelerating voltage between 10 and $25 \mathrm{kV}$ and magnification of up to $1000 \times$. In addition, energy dispersive analysis was performed to investigate the elemental composition of filler particles. This characterization was carried out on the cross-sectioned, mounted, and polished free film samples. Lastly, to gain better insight into the distribution of defects inside the coating films, without exposing them to water during wet polishing, free film samples were immersed in liquid nitrogen for about $20 \mathrm{~s}$ and then broken to prepare cross-sections for micromorphology observations. For all SEM examinations, compressed clean air was used to remove debris from samples, and the prepared cross sections were sputter-coated with a thin gold-palladium layer to provide conductivity.

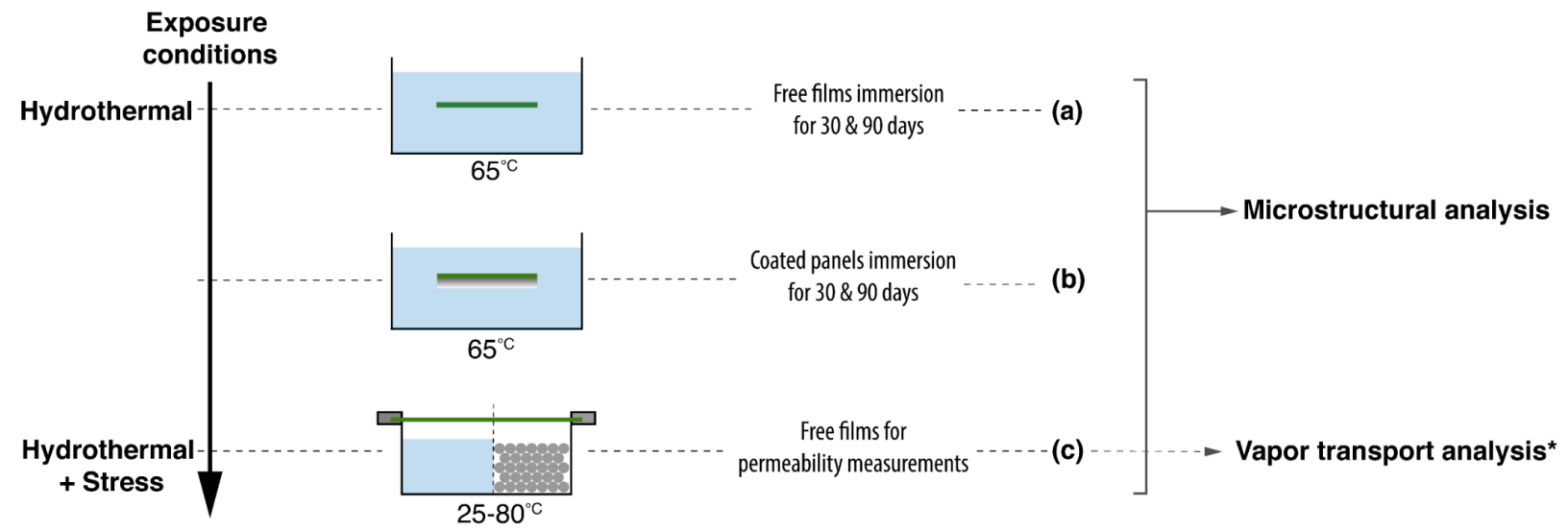

Figure 1. Schematic overview of the water ingress analysis for epoxy-based powder coatings

* Measurements were conducted using molecular sieves and deionized water for dry- and wet-cup conditions, respectively.

\subsection{Water vapor permeability}

To determine water permeability, we applied gravimetric cup methods based upon protocol ASTM D165303 (i.e., c in Figure 1) [30]. Vapometer cups, consisting of a container made of a noncorroding material impermeable to water, were used to measure the steady water vapor flow in unit time through unit area of a film under specific conditions of temperature and humidity at each face of the film. Exposure conditions were modified to measure vapor transmission above the standard temperatures. Dry cups contained fresh 
molecular sieve desiccant and wet-cups contained DI water to establish $0 \%$ and $100 \%$ relative humidity $(\mathrm{RH})$, respectively, on one face of the coating sample. Each test specimen was sealed against the open mouth of a Vapometer cell (No. 68-3000, Thwing-Albert Instrument Co., West Berlin, NJ, USA) and the assembly was placed in the test chamber with a controlled atmosphere. The geometrical test area of the films was $31.67 \mathrm{~cm}^{2}$, and the mass of the cup was sequentially recorded over 12 days using an automated microbalance (with $1 \mathrm{mg}$ sensitivity), allowing the effective permeability to be derived from the slope of the steady state mass change data over time. Test chamber temperatures were $25,40,65,70$, and $80^{\circ} \mathrm{C}$ and $\mathrm{RH}$ was generally maintained at $50 \%$. For $\mathrm{FBE}$ films, tests at $65^{\circ} \mathrm{C}$ were piloted in several $\mathrm{RH}$ conditions $(10,30$, and $50 \%)$ to investigate the effect of water activity on vapor transport. As per ASTM D1653, temperature and humidity inside the chamber were regulated at $\pm 1^{\circ} \mathrm{C}$ and $\pm 2 \%$, respectively. Test conditions were also monitored externally with an EL-USB-2 temperature / humidity data logger (Lascar Electronics Ltd., PA, USA). Periodic weighing of cups was made in a few seconds to minimize errors due to internal pressure variations throughout each test and permeability data were taken as an average of three measurements (at minimum) for each test condition.

Based on the weight changes of each cup, we plotted the vapor transport flux against elapsed time. Vapor transmission rate or the normalized concentration-based permeability $\left(P_{L}\right.$ in $\left.\mathrm{mol} / \mathrm{m}-\mathrm{s}\right)$ and gas phase permeability $(P$ in $\mathrm{mol} / \mathrm{m}-\mathrm{s}-\mathrm{Pa})$ were calculated using:

$$
P=\frac{P_{L}}{\Delta p}=\frac{J . l}{A\left(p_{f}-p_{s}\right)}
$$

where $J(\mathrm{~mol} / \mathrm{s})$ is the vapor flux resulting from the slope of the regression line, $l(\mathrm{~m})$ is the coating thickness, $A\left(\mathrm{~m}^{2}\right)$ is the test area, $p_{f}$ and $p_{s}$ are absolute water pressure $(\mathrm{Pa})$ on the vapor feed and vapor sink, respectively. Vapor pressures were derived from saturation pressure of water at the test temperature [32] times RH conditions (as decimals) at feed and sink sides.

\subsection{Transport properties of the multilayered HPPC}

HPPC is a composite membrane that has a configuration of a sealing (or protective) layer, a selective layer, and a support substrate which usually contains porosity - i.e., a polyethylene topcoat followed by a 
polyolefin adhesive and then an FBE primer [33], [34]. Vapor transmission through a defect-free laminate from different polymeric layers is expected to follow the ideal laminate theory [35]. Thus, WVT results for FBE and polyethylene topcoat samples are anticipated to yield a dependable estimate for $P_{L}$ through an HPPC film - this assumes that the adhesive and topcoat layers are single PE layer. The permeability coefficient for a multilayer membrane $\left(P^{H P P C}\right)$ is a function of the thickness $\left(l^{i}\right)$ and permeability $\left(P^{i}\right)$ of constituting layers, where:

$$
\frac{P^{H P P C}}{\sum l^{i}}=\frac{1}{\sum l^{i} / P^{i}}
$$

Validity of this equation for HPPC is typically studied at $65^{\circ} \mathrm{C}$ for WVT tests [5]. Due to unclear contribution of the constituting layers to the mass transfer resistance of the multilayered HPPC, we used permeance $\left(\mathrm{mol} / \mathrm{m}^{2}-\mathrm{s}-\mathrm{Pa}\right)$ instead of permeability to assess the coating performance from direct empirical results, in which:

$$
\text { Permeance }=\frac{J^{H P P C}}{A \Delta p}=\frac{P^{H P P C}}{\sum l^{i}}
$$

where $\Delta p$ is the pressure difference across the multilayered membrane as defined in Equation (1). Unlike permeability, the permeance is strongly dependent on the thickness of the membrane and driving force (e.g., upstream and downstream pressures); however, comparisons of permeance data are still expected to reflect variations in flux due to vapor-liquid equilibrium effects. In other words, although permeance can change with feed pressure similar to flux, it does not do so as markedly, and analyses of transport based on permeance can provide insightful information [36].

\section{Results and Discussion}

\subsection{Water transport analysis of FBE}

We plotted vapor transport isotherms of FBE using a nonlinear regression analysis for different $\mathrm{RH}$ conditions at $65^{\circ} \mathrm{C}$ (Figure 2). Our results showed that the vapor permeance of the FBE film was sensitive to humidity limits and measurements from the cup methods were not equal. This behavior, which has been noted in the literature [37], was an early indication of hygroscopic behavior in the coating. Since data from 
dry- and wet-cup measurements were generated during exposure to lower and higher water vapor concentrations, respectively, higher absorption of water made the coating less dense, allowing moisture to transport at relatively higher rates [30]. At lower activities (e.g., measured data in dry-cup limits), water transmission rate increased with concentration close to a linear manner because the Langmuir and Henry's sorption modes, also known as dual-mode sorption, are dominant processes [38], [39]. Although we generally expect a linear relationship between permeability and relative feed pressure, gas permeation based on dual mode transport is described by an exponential expression [40], [41]. We found a small nonlinear relationship between the water vapor transmission rate (i.e., $P_{L}$ ) and its concentration. The effect of water activity on transmission through FBE was also notable when the concentration gradient was high in either dry- or wet-cup isotherms. The non-linear trend suggests a transition in sorption or diffusion in the polymer network, which is often attributed to self-association of water inside the polymer [39], [42]. The hole-filling mechanisms (i.e., Langmuir and Henry's type sorption) reach an equilibrium above $\sim 0.5$ water activity, and a dynamic water clustering occurs in the glassy polymeric network-activation of pooling mode sorption [42]. That being said, the slight drifts from linearity for wet-cup limits in Figure 2 suggest the formation of less water clusters within FBE network as compared to other hygroscopic glassy polymers [43]. 


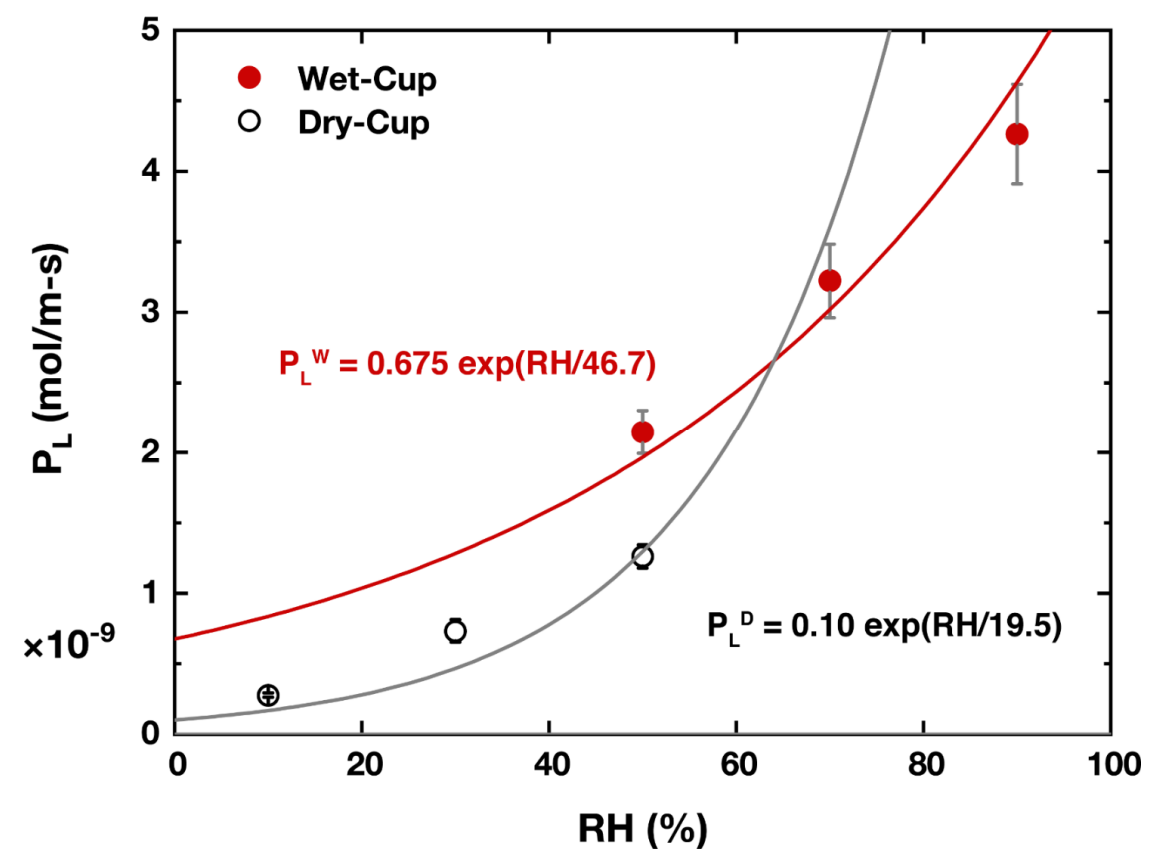

Figure 2. Vapor transmission results at $65^{\circ} \mathrm{C}$ for vapometer cups with internal relative humidity of $0 \%$ and $100 \%$ (i.e., dry- and wet-cup limits, respectively) in external relative humidities of 10,30 , and $50 \%$. Relative humidity inside the chamber was calculated as the ratio of the actual water vapor pressure to the saturated water vapor pressure of the air at the test temperature. Three replications were made for each test condition. Each data point is an average +/- standard deviation. Grey and red solid lines were obtained from extrapolation of $P_{L}$ in dry- and wet-cup conditions $\left(P_{L}^{D}\right.$ and $P_{L}^{W}$, respectively).

Permeability analysis of the WVT data in Figure 2 showed little contrasts between permeabilities for each FBE isotherm - i.e., $10^{-13}$ and $1.8 \times 10^{-13} \mathrm{~mol} / \mathrm{m}-\mathrm{s}-\mathrm{Pa}$ on average from dry- and wet-cup measurements, respectively (Figure 3). The small difference between data within each humidity limit is rooted in approximation of the steady-state flow across films according to boundary conditions. Indeed, these values represent the average permeability associated with the dry- and wet-cup limits [44]. Previous work in the literature [37] has shown that these average permeabilities can be applied as baselines for constructing spot permeability curves (solid line curves in Figure 3). According to the equations governing steady state permeability in glassy polymers under humified conditions [41], [45], the spot permeability should be expressed as an exponential function of vapor activity. In this study, we applied the Chang and 
Hutcheon method [44] to extract curves of spot permeability from empirical dry- and wet-cup data. Each curve generates two equal areas within its designated limits (triangular areas in Figure 3). The resultant area between the two curves provides valid approximates for average permeability in general cases outside the tested limits. For instance, when an a priori dry FBE coated steel pipe is in service at $65^{\circ} \mathrm{C}$ with an average $\mathrm{RH}$ value of $70 \%$, one can use reference equations for $P_{D}$ and $P_{W}$ to estimate a range for the nominal permeability value within 0 and $70 \% \mathrm{RH}-$ i.e., by finding a range in which equal areas are generated between the dry (and wet) curve and these RH limits.

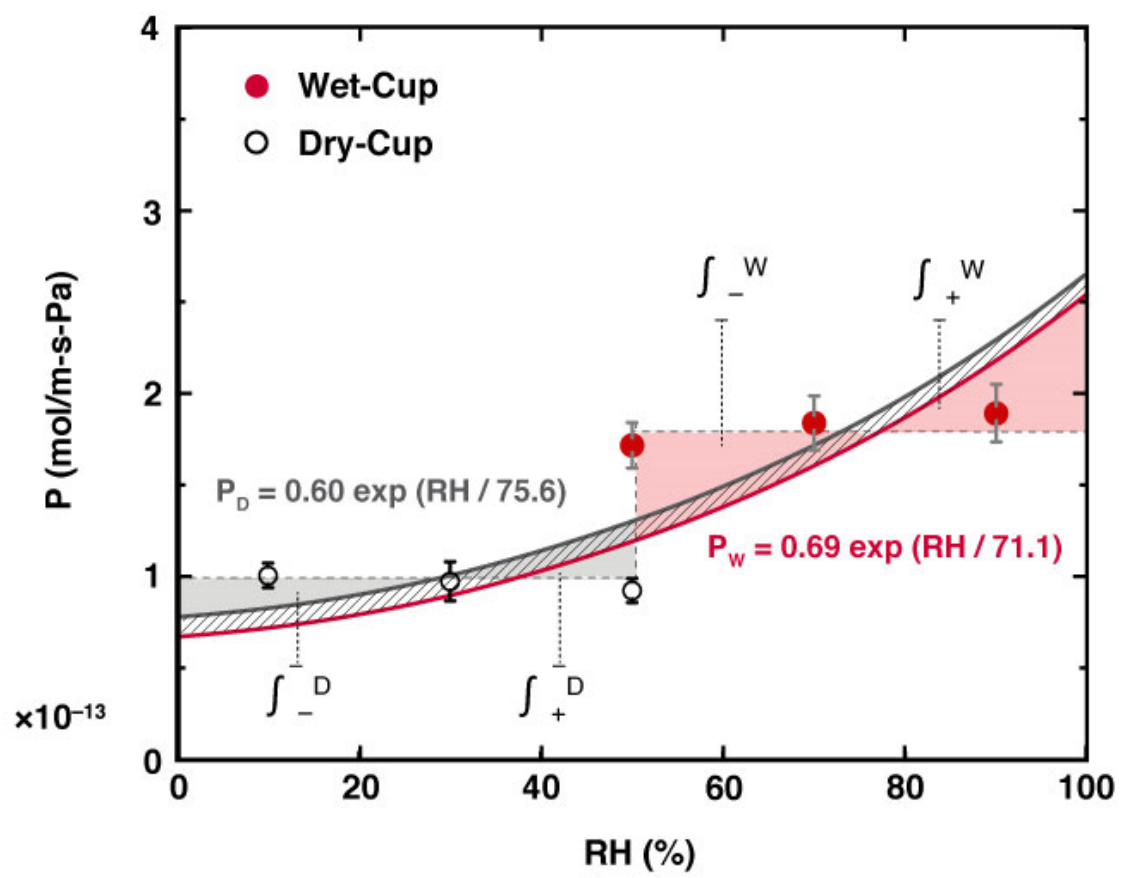

Figure 3. Average permeability data at $65^{\circ} \mathrm{C}$ versus relative humidity and derivation of spot permeability curves for vapor transport (based on the Chang and Hutcheon method [44]). Each data point is an average +/- standard deviation. The spot permeability curves were obtained using a Brute-force integration over the two cup method results $\left(\left|\int_{-}^{D}-\int_{+}^{D}\right| / \int_{-}^{D}>2 \times 10^{-8} \&\left|\int_{-}^{W}-\int_{+}^{W}\right| / \int_{-}^{W}>7.5 \times 10^{-8}\right)$.

To further verify and validate the nominal permeability curve in Figure 3, one can fit the experimental data with mathematical models based on the dual mode sorption theory (see [45]). Such an analysis, which 
requires fugacity-based sorption parameters of water in epoxy, can be the focus of a future study. Our results showed approximately one order of magnitude less permeability for water vapor than that reported previously by Fu and Cheng [46] - e.g., their analysis shows $P_{F B E}=18 \times 10^{-13} \mathrm{~mol} / \mathrm{m}$-s-Pa for a wet-cup measurement at $65^{\circ} \mathrm{C}$ while it was $2 \times 10^{-13} \mathrm{~mol} / \mathrm{m}$-s-Pa at similar conditions in this work. This may be due to different FBE formulations, or it may reflect these authors applying an imperfect sealing system in their cup method (i.e., using "water-proof epoxy resin" as a sealant). The data presented herein are in good agreement with results published in other literature for epoxy materials [5], [47]. Our study indicates that although measuring water vapor transmission rate and permeability at high temperatures can significantly vary with water activity, cup methods, if carefully implemented, can generate data with excellent confidence. Also, our quantification of water permeability versus vapor activity (i.e., spot permeability curves in Figure 3) can be used as a basis for modeling permeability parameters for FBE (i.e., the infinite dilution diffusivity, immobilization factor, and plasticization potential) in future studies [41].

The effect of thermal conditions on water transport also showed a nonlinear increase of vapor transmission rates (i.e., vapor flux) as a function of temperature, ending with a significant rise (Figure 4). The final increase in $P_{L}$ indicates that plasticization of the FBE occurs once the critical concentration of water within epoxy is exceeded during the temperature elevation, in other words when plasticization pressure is well below the vapor saturation pressure at $80^{\circ} \mathrm{C}$. The difference between wet- and dry-cup results reached up to one order of magnitude at the upper limit, showing the extreme effect of vapor feed pressure on the transport process. Such a distinction at one test temperature also confirms that, at lower activities (drycup limits), water molecules are bonded at polar sites in the epoxy network and have less tendency for outgassing from the polymeric membrane. Previous work also showed that translational and rotational movements of water molecules within the epoxy are hindered by the glassy and stiff structure at low concentrations [48]. According to Flory-Huggins theory [49]-[51], the interaction between a solvent (e.g., water) and a polymer is temperature dependent. This correlation helps explain why a narrow, almost negligible, margin exists between results from dry- and wet-cup tests at low temperatures $\left(25\right.$ and $40^{\circ} \mathrm{C}$ in Figure 4). One must note that conditioning of the glassy polymer at these low temperatures enables the 
bonded water molecules to act as a nucleus for cluster formations [48]. By increasing temperature, the endothermic microvoid formation (or clustering) develops in the sorption process, and the associated irreversible process initiates microdamaging and degradation of the epoxy network [39].

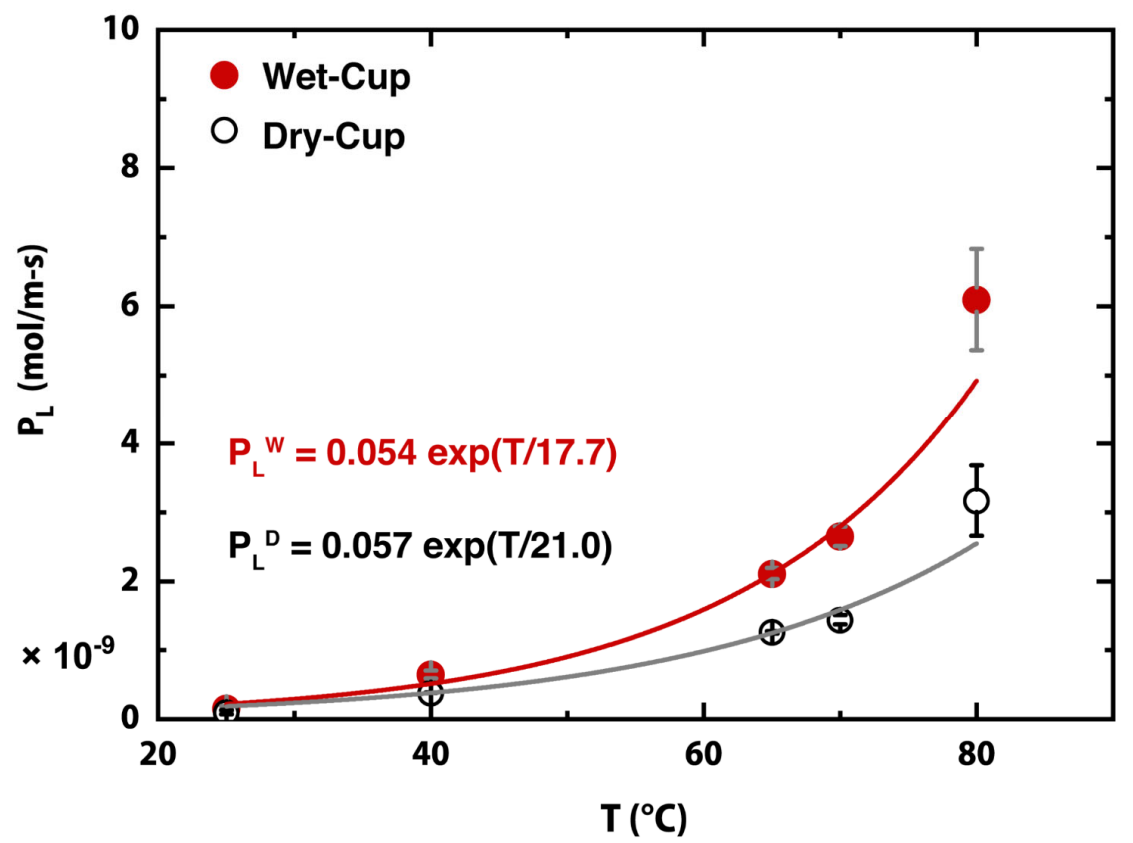

Figure 4. Water vapor transmission through fusion-bonded epoxy samples vs. temperature. Each data point is an average $+/$ - standard deviation. Grey and red solid lines were obtained from extrapolation of $P_{L}$ in dry- and wet-cup conditions $\left(P_{L}^{D}\right.$ and $P_{L}^{W}$, respectively); curves represent exponential regression analysis with probability values less than 0.005 .

In general, gas phase permeability is an insubstantial function of concentration and temperature and does not follow an exponential growth similar to $P_{L}$ [36]. In our study, changes in the average permeability of FBE at different temperatures, however, showed the synergistic effect of high temperature and pressure on vapor transport (Figure 5). Permeability initially increased as temperature was raised to $40^{\circ} \mathrm{C}$, then followed a small declining trend in the middle range, but eventually turned up and began to increase due to plasticization effects. According to the van't Hoff-Arrhenius model, the temperature dependency of permeability through a polymer at a constant pressure is described by an energetic parameter and a pre- 
exponential factor $\left(E_{P}\right.$ and $P_{0}$ in $P=P_{0} \exp \left(-E_{P} / R T\right)$, respectively [52]). This model predicts a progressive increase of permeability versus temperature in Figure 5. On the other hand, the pressure dependency of permeation through glassy polymers is a function of permeant/polymer interactions and one may encounter different qualitative trends by increasing feed pressure [53]. Generally, an initial decrease in permeability is followed by an increasing drift after a plasticization pressure, as described by the dualmode sorption model [54], [55]. Accounting for the effect of temperature on the vapor pressure in Equation (1), the permeability data, as shown in Figure 5, are in agreement with previous findings on glassy polymers, which explain the gas permeability based on the predictions of the dual-mode sorption theory [54]. Additionally, the slight decrease of permeability between 40 and $65^{\circ} \mathrm{C}$ in Figure 5 was also in accord with reports of water clustering effects in epoxy above $45^{\circ} \mathrm{C}$, where the 'water-induced microvoid' situation became kinetically favorable due to its endothermic nature [48].

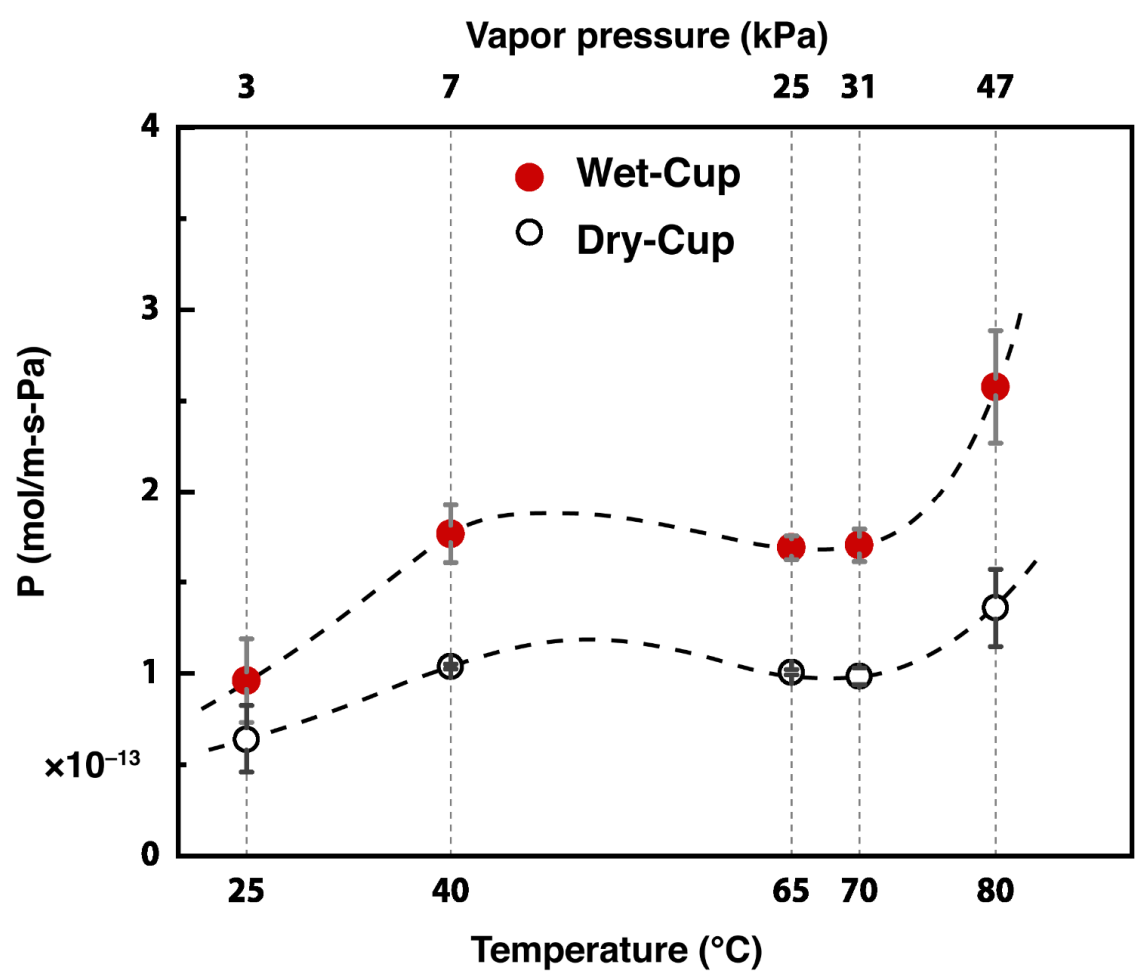

Figure 5. Water vapor permeability of fusion-bonded epoxy at different test temperatures. Each data point is an average $+/$ - standard deviation. The dashed lines are for guidance only. 
Previous work in the literature [56] has shown through thermodynamic characterization of water clusters that formation of a more ordered structure from dissolved water has large enthalpy gain and entropy loss, resulting in a slightly negative $\Delta G$. Specifically, the thermodynamically metastable state of water due to clustering slows the transmission rate through the polymeric network, resulting in a significant decrease of diffusivity of water molecules in the highly ordered water clusters. Upon further temperature increase (above $70^{\circ} \mathrm{C}$ ) in our experimental design, the FBE network was plasticized by vapor permeant and concentration (or pressure) of the permeant became a determining factor in the barrier performance of the coating. In other words, although the clustering effect was likely to decrease permeability of the coating membrane, and therefore increase the selectivity over other permeants, the role of a permeant concentration is expected to become more pronounced for the hydrated polymer at elevated temperatures [47]. The occurrence of minimum permeability at $\sim 70^{\circ} \mathrm{C}$ is consistent with the observations of other researchers in glassy polymeric membranes [41]. This transition in permeability is thought to mainly relate to the break-up of water clusters at this temperature due to lower water concentrations in the polymer. The following increase in permeability is also a result of higher diffusivity of individual water molecules than water clusters at higher temperatures [39].

\subsection{Microstructural degradation of FBE upon hydration}

The cross-sectional morphologies of FBE film samples were examined by SEM; an example of a resulting image of an FBE coating is presented in Figure 6. With the energy dispersive X-ray analysis, we found acicular particles of calcium silicate dispersed within the FBE microstructure. The spectrum of the mapped area also indicated traces of magnesium and aluminum (below 7 and 2 mass $\%$, respectively). Previous work has confirmed that the calcium silicate-rich fillers are needle-shape amorphous wollastonite $\left(\mathrm{CaSiO}_{3}\right)$, which is a functional filler added to increase flexural modulus and reduce the thermal expansion and shrinkage of the final coating [57]. Sugiman et al. demonstrated that adding micro-fillers into the epoxy resin decreases the crosslink density, subsequently increases the swelling strain during water transport [58]. On the other hand, increasing the volume fraction of fillers may subject the polymer to a percolation effect for water diffusion; a critical volume fraction (percolation threshold) of $p_{c}=0.157$ is reported for 
epoxy composites [59]. For an average volume fraction of $0.074 \pm 0.01(0.037-0.12)$ inorganic content in the resin, with manufacturer specifications in parentheses, the FBE tested here would not be anticipated to experience a fast water uptake as a result of segregation of the absorbed water from the polymer chains [58]. High activities of water (> 0.8), however, have been shown to induce minor water clustering in epoxy systems similar to FBE [60]. If the filler is reactive with water, the time for reaching the saturation would increase, as diffusion rate would decrease, and water transport would be hindered due to the resulting trapping mechanism [29].

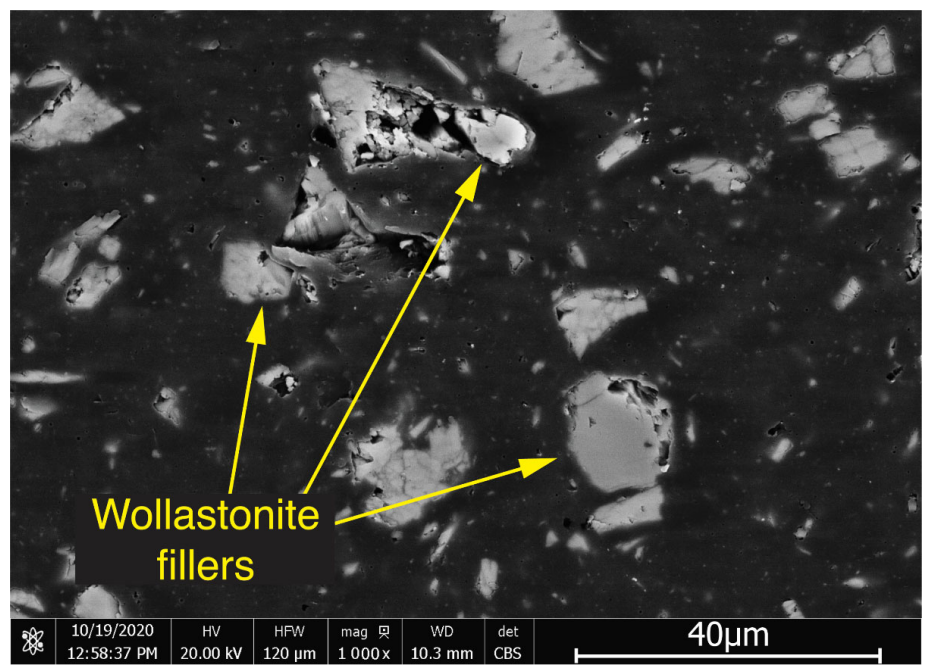

Figure 6. Back-scattered electron image of cross-sectional morphology of fusion-bonded epoxy microstructure; energy dispersive X-ray analysis of filler particles indicates presence of $\mathrm{Ca}$ and $\mathrm{Si}$ in high quantities ( $>45 \%$ and $>40 \%$ atomic mass, respectively).

In our microstructural analysis, we observed pre-existing porosity defects in FBE coatings, and their size and distribution varied with the history and conditions of the coating application. According to the CSA Z245.20-18 standard released in 2018 [61], a coating is considered qualified if its associated porosity is lower than a certain limit; five rating scales are graphically defined for cross-section porosity and a coating should include equal to or less than that of rating three to be approved. Thus, unlike three-layer polyethylene systems in which no air entrapment is allowed in the topcoat layer [62], [63], the FBE coating may contain allowed cavities within its microstructure. The size and distribution of associated cavities are 
related to preheat temperature of the substrate, powder density of the spraying stream per pass applied and a possible minor contribution from evaporation of water/solvent compounds throughout the epoxy curing process [64].

We generated back-scattered electron (BSE) images from different water exposure conditions to show the extent of the damage of both immersion and WVT tests to the FBE films (Figure 7). Long-term exposure of $\mathrm{FBE}$ to $65^{\circ} \mathrm{C} \mathrm{DI}$ water affected the interfacial bonds between epoxy resin and filler particles. As can be seen in Figure 7a, segregation of wollastonite particles from the epoxy-rich phase was distinct from unaged FBE (i.e., Figure 6). Upon addition of stress to the hydrothermal conditions (i.e., as in WVT films), shear deformation of the epoxy network was likely to occur, which would eventually cause microcracking and cavity formation (Figure $7 \mathrm{~b}$ ). It is pertinent to note that the morphology of the coating microstructure represented by the polished samples might yield an arbitrary representation of defects in the polymeric network. To further qualify the degradation, FBE free films were cryogenically sectioned and examined via SEM imaging (Figure $7 \mathrm{c}$ and d). When we compared the fracture surfaces of an unaged film sample and a film tested at a dry-cup assembly at $65^{\circ} \mathrm{C}$, we found that coating degradation not only led to microcrack formations inside the epoxy, but it also resulted in less bonding between matrix and filler particles - wollastonite particles were highly incorporated in the epoxy resin in Figure 7c, whereas they were dispersed on the fracture surface in Figure $7 d$. Although water absorption by epoxy tends to be a partially reversible process, especially for the polymer's response to mechanical loading [65], microstructural damage caused by plasticization effects are irreversible [5], [66]. Therefore, we did not examine the reversibility of microcrack formation in this study. It appears that hydrothermal exposure can cause excessive tearing and remarkably increase nonselective channels within the membrane. In a protective coating, such a deterioration can result in Knudsen flow (molecule-pore wall collisions) of penetrant molecules, and therefore poor barrier performance. The newly formed domains are taken up by water molecules in wet systems and associated clustering decreases the diffusivity (ergo the permeability) of permeants including water itself [67]. The polymeric coating, however, has already been subjected to 
severe damage and its barrier properties can significantly decline as soon as plasticization effects evolve at higher permeant temperatures or pressures [68].
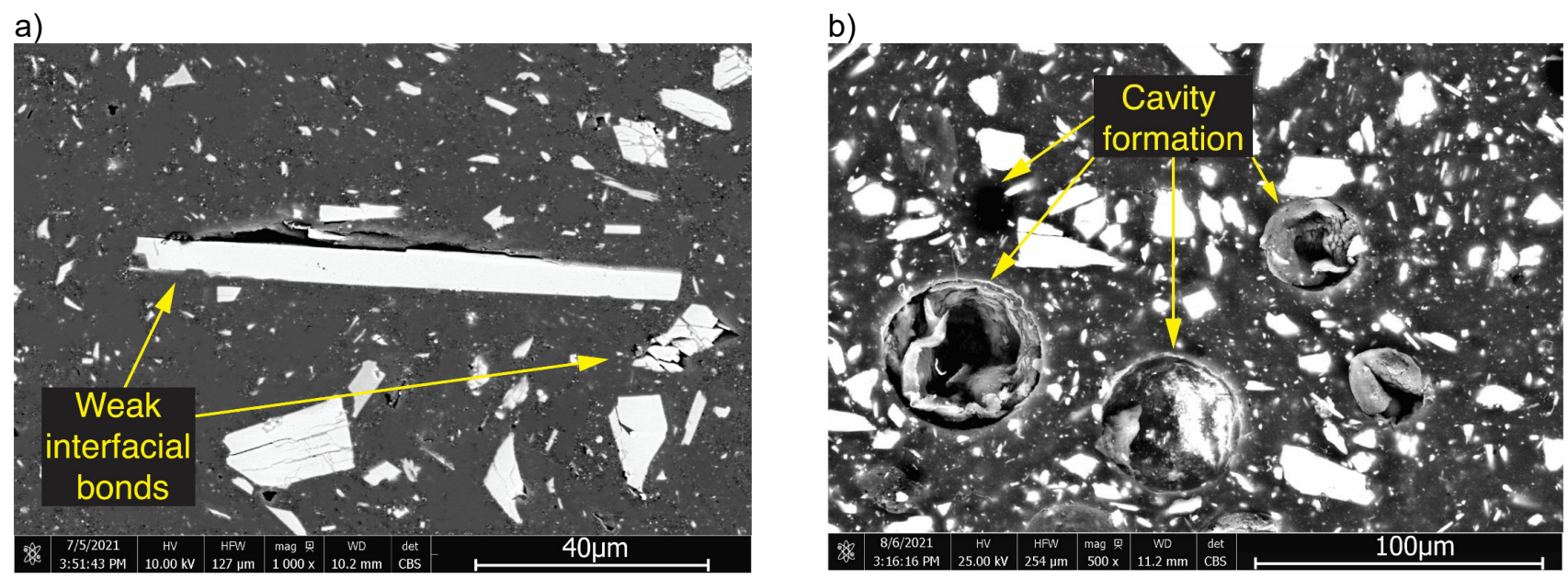

c)

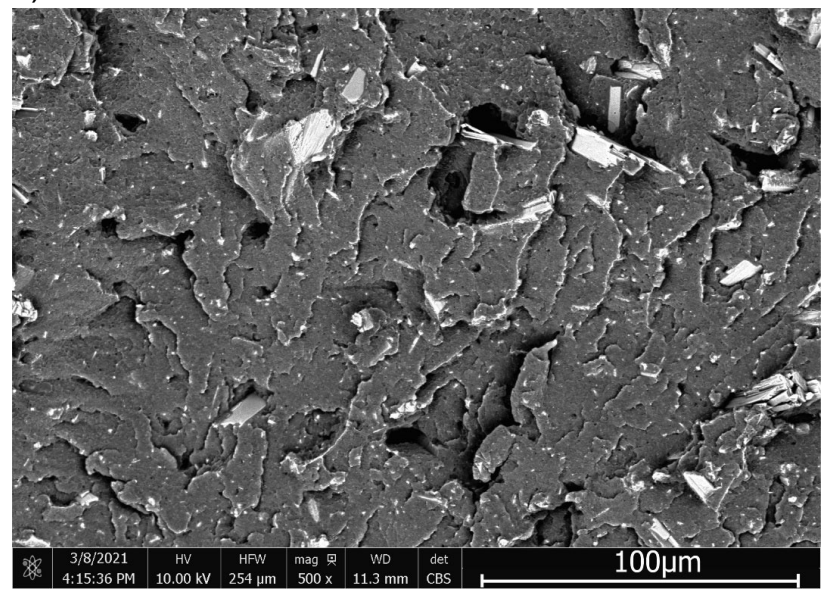

d)

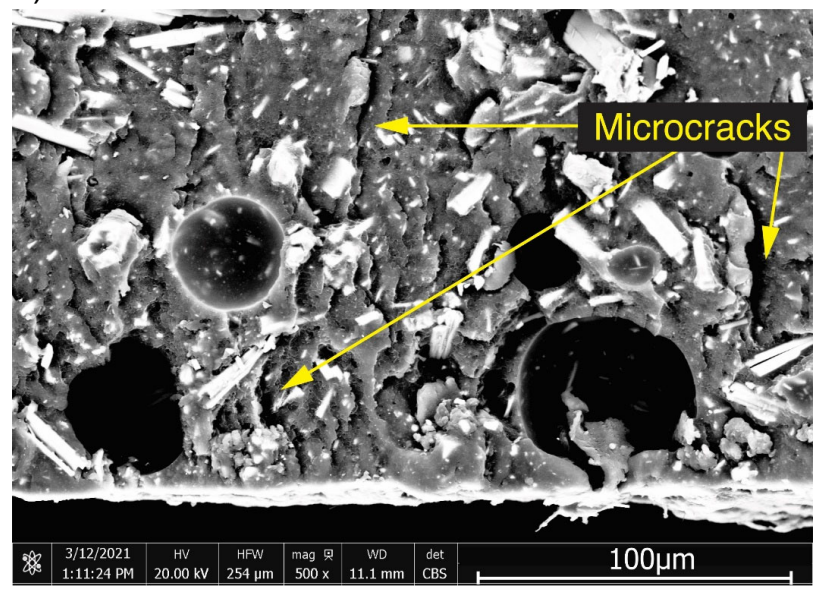

Figure 7. Micrographs of fusion-bonded epoxy: a) after exposure to $65^{\circ} \mathrm{C}$ deionized water for 90 days; b) after dry-cup water vapor transmission test at $65^{\circ} \mathrm{C}$; c) cryogenic featuring of an unaged film; d) cryogenic featuring of film after dry-cup water vapor transmission test at $65^{\circ} \mathrm{C}$.

According to both computational and experimental analyses of pipeline coatings, the energy associated with FBE that is applied on a steel substrate during manufacturing generates internal stresses inside the coating due to different thermal contraction coefficients between steel and thick (i.e., above $250 \mu \mathrm{m}$ ) polymeric coatings [19]. Comparative studies on epoxy-free films and coatings during hygrothermal ageing confirm that diffusion kinetics increase with temperature (from $30^{\circ} \mathrm{C}$ to $60^{\circ} \mathrm{C}$ ) and that diffusivity depends 
upon the microstructure (such as the polar group concentration and the free volume fraction) [43]. Although the polymer structure is locally modified in the applied coating and contains different diffusional pathways compared to free films, the relaxation of internal stresses in the applied coating during sorption is expected to decrease the diffusion rate close to the free film values [43]. Our SEM analysis indicated that the extent of polymer degradation in both free films and applied coatings was consistent and that long-term hydrothermal exposure to water at $65^{\circ} \mathrm{C}$ could lead to significant changes in porosity of applied FBE microstructure (Figure 8). We examined two different FBE panels for this analysis and compared the effect of hydrothermal exposure on specimens from these panels after 30 and 90 days. According to CSA Z245.20-18 [61], these panels were initially classified as rating of one and three (Figure 8a and c, respectively). After 30 days hydrothermal exposure, the coating microstructure was indicative of small cavity formations in the epoxy resin (Figure 8b compared to 8a). These micropore formations can induce further plasticization of the coating profile upon subsequent sorption of water. In other words, cross-section porosity present in the coating can increase during water permeation and, for instance, an unaged coating with the initial rating of three is highly likely to be classified as four or five after hydrothermal exposure (Figure $8 \mathrm{c}$ and d). Although clustering of absorbed molecules inside polymer reduces diffusional mobility inside epoxy upon formation of micropores, larger holes are expected to increase the solubility of the permeating water [69]. This degradation results in loss of selectivity of the coating, since transport behavior of the polymer can be highly dependent upon kinetics of water sorption/desorption processes within the polymer [5], [43]. In addition, appearance of interfacial disbondments of FBE was also notable in Figure $8 \mathrm{~d}$, which indicated that adhesion loss and corrosion became thermodynamically favorable in the system (mass flux through coating correlates with transient occurrence of underlying corrosion reactions). This is of significance since immobilization of permeant at fixed sites within the polymeric medium, in microvoids or holes in the FBE structure, is highly temperature-dependent [28], and the gas held by these holes might be only partially immobilized [70]. 


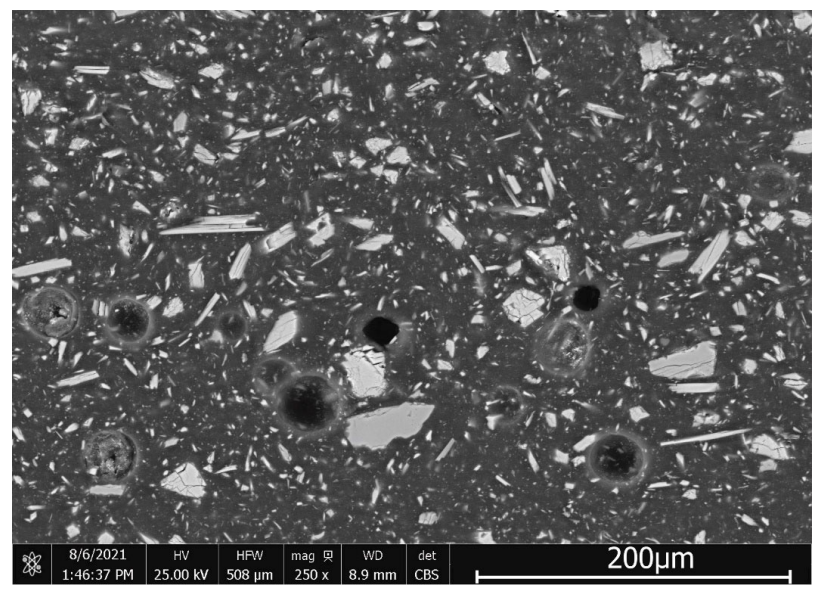

c)

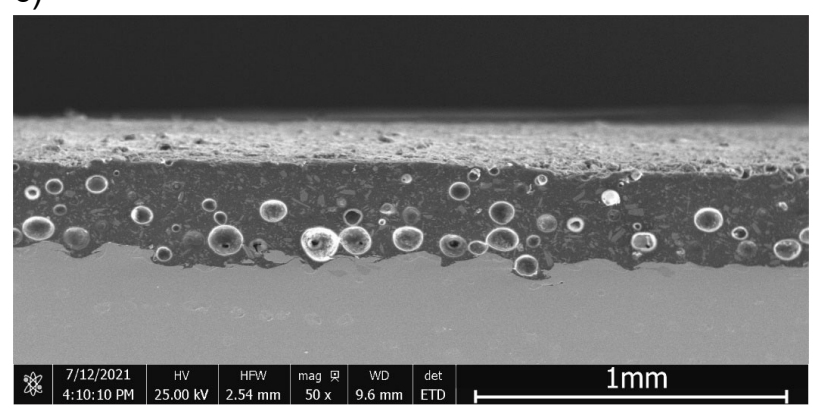

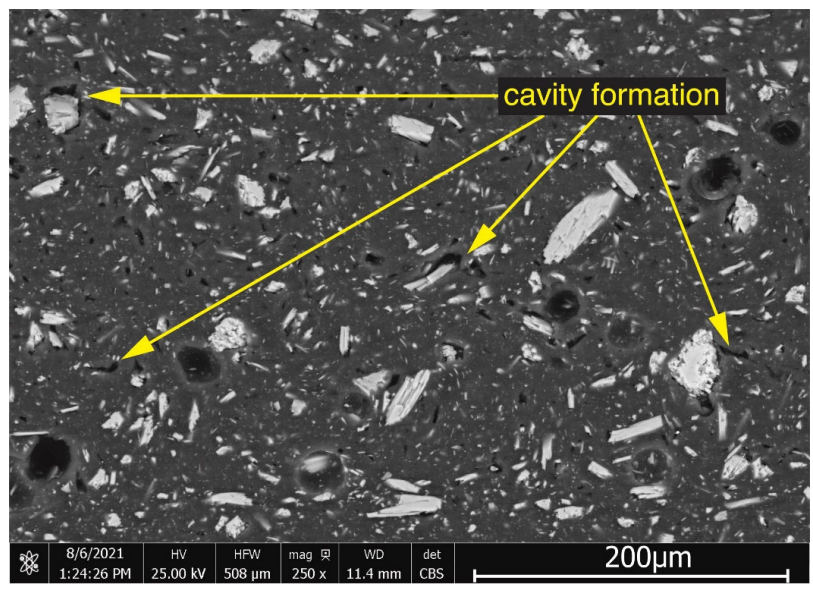

d)

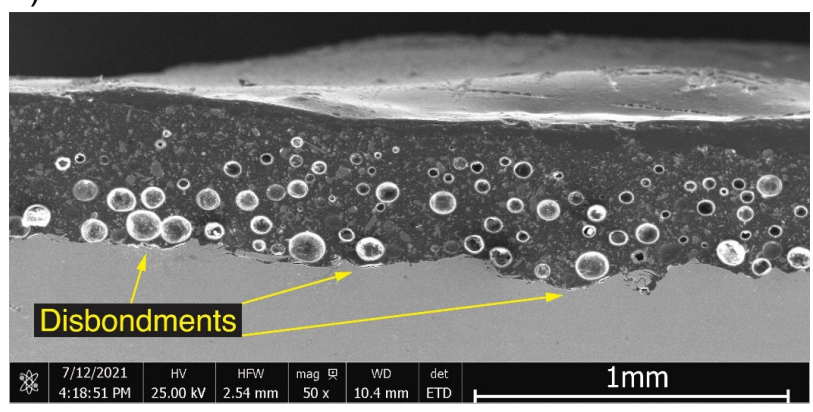

Figure 8. Micrographs of fusion-bonded epoxy panels: a) back-scattered electron image of an unaged specimen (rating of one); b) back-scattered electron image after immersion in $65^{\circ} \mathrm{C}$ deionized water for 30 days (initially classified as rating of one); c) secondary electron image of an unaged specimen (rating of three); d) secondary electron image after immersion in $65^{\circ} \mathrm{C}$ deionized water for 90 days (initially classified as rating of three). CSA Z245.20-18 has been used as the classification criteria for the assessment of porosity in test specimens.

\subsection{Vapor permeance through topcoat $P E$ and HPPC}

Vapor transmission rates through topcoat PE films at ambient temperatures ( 25 and $40^{\circ} \mathrm{C}$ ) were outside the accuracy limit of the assembled WVT setup. For instance, a weight loss of $\sim 0.04 \mathrm{mg} /$ day is expected for a vapometer cell mounted with the topcoat PE sample at $25^{\circ} \mathrm{C}$ [62]. Therefore, for vapor transmission through topcoat PE and HPPC, the major focus was on measurements at elevated temperatures. Table 1 shows WVT results for topcoat PE films at different temperatures. As expected, both $P_{L}$ and $P$ increased 
linearly with temperature, and no significant contrast existed between data from different humidity limits, which conforms to anticipated properties of polyolefins.

Table 1. Permeability measurements for topcoat polyethylene

\begin{tabular}{|c|c|c|c|c|}
\hline \multirow[t]{3}{*}{ Test condition } & \multirow{3}{*}{$\begin{array}{l}\text { Temperature } \\
{ }^{\circ} \mathrm{C}\end{array}$} & $P_{L}^{*}$ & \multirow{2}{*}{$\begin{array}{l}\text { Permeance } \\
\qquad\left(\times 10^{11}\right)\end{array}$} & \multirow{2}{*}{$\begin{array}{l}\text { Permeability } \\
\qquad\left(\times 10^{14}\right)\end{array}$} \\
\hline & & $\left(\times 10^{10}\right)$ & & \\
\hline & & $\mathrm{mol} / \mathrm{m}-\mathrm{s}$ & $\mathrm{mol} / \mathrm{m}^{2}-\mathrm{s}-\mathrm{Pa}$ & $\mathrm{mol} / \mathrm{m}-\mathrm{s}-\mathrm{Pa}$ \\
\hline & 65 & $4.55 \pm 0.15$ & $4.84 \pm 0.37$ & $3.63 \pm 0.12$ \\
\hline \multirow[t]{3}{*}{ Dry-cup } & 70 & $6.49 \pm 1.05$ & $5.52 \pm 1.04$ & $4.16 \pm 0.96$ \\
\hline & 80 & $12.3 \pm 0.75$ & $7.36 \pm 0.59$ & $5.19 \pm 0.32$ \\
\hline & 65 & $4.66 \pm 0.41$ & $4.82 \pm 0.52$ & $3.72 \pm 0.33$ \\
\hline \multirow[t]{2}{*}{ Wet-cup } & 70 & $6.59 \pm 0.06$ & $5.47 \pm 0.01$ & $4.23 \pm 0.04$ \\
\hline & 80 & $13.3 \pm 1.06$ & $7.75 \pm 0.60$ & $5.62 \pm 0.45$ \\
\hline
\end{tabular}

${ }^{*}$ The normalized concentration-based permeability, as defined in Equation (1)

The projected permeance and experimental results for HPPC samples at $65^{\circ} \mathrm{C}$ are outlined in Table 2. Since permeance depends highly on the film thickness, and since contributions of constituting layers in mass transport were not clear for HPPC (Figure 9), the application of ideal laminate theory (Equation 2) may not provide an effective assessment for mass transport analysis. Table 2 also shows that contrary to the results obtained from FBE, weight changes of dry-cup samples were slightly higher than that of wetcup measurements for HPPC. This anomaly can be related to both the hygroscopicity of the FBE primer upon exposure to higher vapor concentrations and to the tendency of the primer for retaining moisture in wet-cup measurements, this layer was exposed to $50 \% \mathrm{RH}$. Such moisture retention can cause a deviation from the established gradient across the HPPC film in wet-cup conditions. In contrast, in the drycup tests, moisture removal took place somewhat spontaneously, as the FBE primer was exposed to $0 \%$ $\mathrm{RH}$. Although data from the lower $\mathrm{RH}$ range are more relevant for applied coatings, especially following application, wet-cup limit measurements can provide less conservative estimates for pipeline systems in equilibrium with their surrounding wet environments.

Table 2. Permeance data for high-performance powder coating

Test condition Temperature Vapor flux Permeance




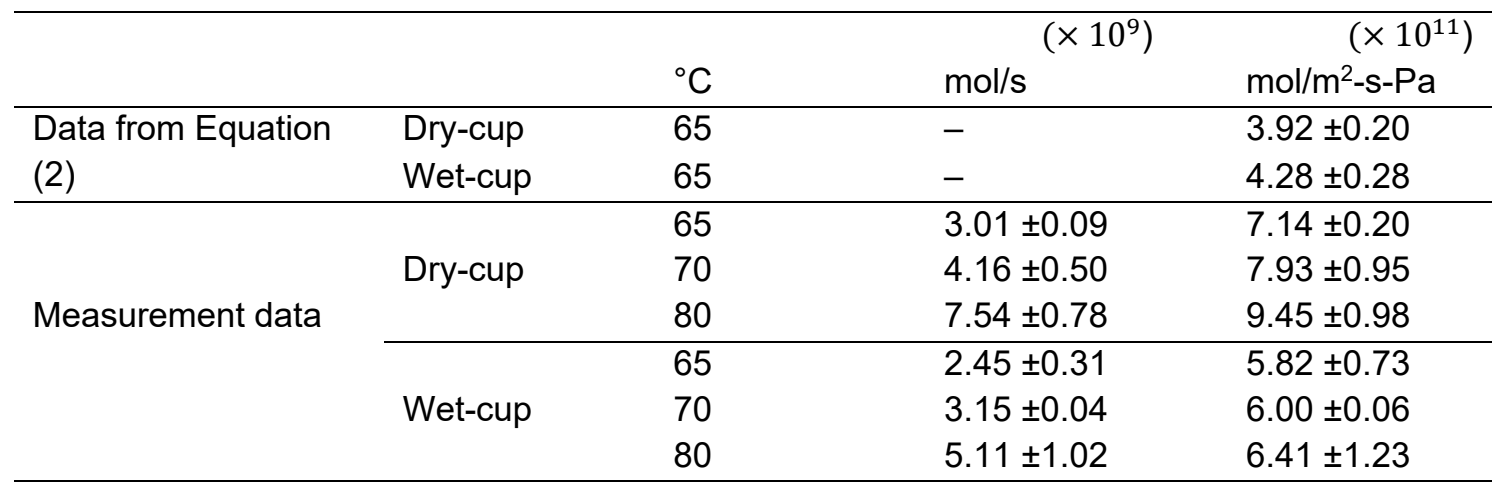

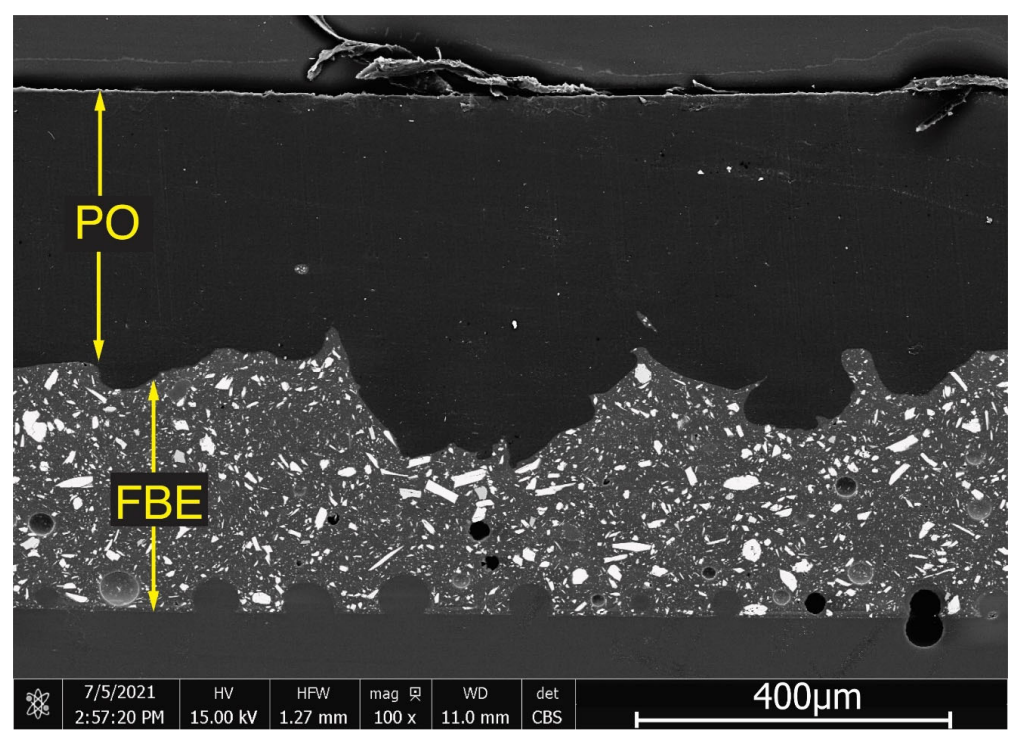

Figure 9. Back-scattered electron micrograph of cross-sectional morphology of high-performance powder coating. PO represents polyolefin adhesive + polyethylene topcoat.

As mentioned above, some of the previously reported data for epoxy coatings fail to accurately assess water permeability at elevated temperature conditions [11], [46]. This argument is further supported by comparing results for HPPC at $65^{\circ} \mathrm{C}$, which showed around a two-order of magnitude higher permeance relative to our data $\left(2.15 \times 10^{-9} \mathrm{~mol} / \mathrm{m}^{2}-\mathrm{s}-\mathrm{Pa}\right.$ for HPPC in [46] compared to data for wet-cup measurement in Table 2). Interestingly, in an earlier work accomplished by the same research group as in [49], a significant vapor transmission rate was reported for the same membrane at room temperature $\left(1.36 \times 10^{-5}\right.$ $\mathrm{mol} / \mathrm{m}^{2}$-s at $23^{\circ} \mathrm{C}$ in [11] compared to $5.4 \times 10^{-5} \mathrm{~mol} / \mathrm{m}^{2}$-s at $65^{\circ} \mathrm{C}$ in [46] for HPPC under identical humidity 
conditions). This shows that misleading data may result from measurements on coating films unless a robust sealing system is used during measurements.

Since solubility of water molecules is the main driver of vapor permeability in the FBE structure [47], an additional moisture resistant layer can mitigate detrimental consequences of interactions between water and the FBE. Results from Table 2 indicate that water vapor permeance can be reduced up to an order of magnitude via the multilayering approach. It should also be noted, however, that these data correspond to an upper boundary for the water transport; the coating and substrate adhesion forces mitigate water ingress prior to occurrence of a disbondment failure. On the other hand, such data provide the primary database to study selectivity of the coating membranes when permeation of other aggressive species (salt or gas) is likely [71]. According to theories developed for gas separation and desalination membranes, the selectivity of a single layer membrane and the separation factor for a multilayered membrane are defined by the ratio of permeability and permeance, respectively, for multicomponent diffusion systems [34], [72]. Since water has a smaller kinetic diameter compared to other penetrants, it plays a key role in the barrier performance (i.e., selectivity or separation factor) of the coating. Thus, permeability data presented in this study enable quantitative analysis of the barrier performance of FBE and HPPC coatings [47].

\section{Conclusion}

This work demonstrates the consequences of interactions between water and the epoxy network and addresses the resulting effects on basic attributes of associated coating systems. Knowledge of transport rate through the protective coating is necessary to better predict and analyze the underlying corrosion in humid environments. From a broader materials characterization point of view, we would like to better understand the onset of coating degradation after exposure to hygrothermal conditions. A number of observations were made for the FBE coating system:

- Epoxy hydration resulted in a nonlinear increase of vapor transmission rate versus concentration. This is where description of mass transport becomes more complicated because the process may include plasticization or clustering effects induced by water molecules. For instance, around $65^{\circ} \mathrm{C}$, 
water self-association tended to decrease the transport rate, while the microstructure of the coating exhibited clear evidence of defect formation due to plasticization by water.

- Vapor transport analysis is a function of humidity limits across the coating film. Yet, average values can be utilized to estimate empirical constants for the concentration dependent vapor permeability.

- The interplay between water clustering and diffusivity manifests itself in the critical temperature of $65^{\circ} \mathrm{C}$, where the polymeric structure is susceptible to plasticization but permeability decreases slightly due to the reduction of diffusivity.

- Epoxy hydration above ambient temperatures $\left(>40^{\circ} \mathrm{C}\right)$ would diminish the interfacial adhesion between filler particles and epoxy resin. Under such conditions, if hydrothermal stress (either swelling pressure or internal stress) is added to the system, it can lead to microcracking and void formation in the coating membrane.

The notion that hydrothermal exposure can alter the aggregate porosity of the coating may necessitate a careful review and implementation of industry standards for pipelines and other coated structures that are exposed to hydrothermal conditions. In our study, multilayered HPPC was more resistant toward water ingress and showed transport behavior closer to topcoat PE. The vapor transport reduced by one order of magnitude for HPPC at temperatures where the FBE primer experienced plasticization effects $\left(>65^{\circ} \mathrm{C}\right)$. Empirical data and performance analyses presented in this study can shed light on barrier performance of the coatings of interest at elevated temperature. They can also provide a solid foundation to study selectivity performance of these materials in binary diffusion systems, such as wet gas permeation or salt ingress, which are of significance not only for pipeline applications but also for process equipment and underground storage tanks.

\section{Acknowledgment}

The authors acknowledge funding support from the Natural Sciences and Engineering Research Council (NSERC) of Canada [NSERC CRDPJ 503725-16]. The funding and in-kind support from Shawcor Ltd. and Specialty Polymer Coatings Inc. [Industry portion - NSERC CRDPJ 503725-16] are also greatly 
appreciated. The authors appreciate Mr. Parham Zarei for his valuable suggestions and kind collaboration for generating the brute force script for data analysis.

\section{References}

[1] S. J. Harley, E. A. Glascoe, and R. S. Maxwell, "Thermodynamic study on dynamic water vapor sorption in Sylgard-184," J. Phys. Chem. B, vol. 116, pp. 14183-14190, 2012.

[2] S. J. Harley, E. A. Glascoe, J. P. Lewicki, and R. S. Maxwell, "Advances in modeling sorption and diffusion of moisture in porous reactive materials," ChemPhysChem, vol. 15, pp. 1809-1820, 2014, doi: 10.1002/cphc.201301097.

[3] V. Baukh, H. P. Huinink, O. C. G. Adan, S. J. F. Erich, and L. G. J. Van Der Ven, "Water-polymer interaction during water uptake," Macromolecules, vol. 44, pp. 4863-4871, 2011.

[4] Y. Liu, W. Soer, J. Scheerder, G. Satgurunathan, and J. L. Keddie, "Water vapor sorption and diffusion in secondary dispersion barrier coatings: A critical comparison with emulsion polymers," ACS Appl. Mater. interfaces, vol. 7, pp. 12147-12157, 2015, doi: 10.1021/acsami.5b02446.

[5] E. Linde, N. H. Giron, and M. C. Celina, "Water diffusion with temperature enabling predictions for sorption and transport behavior in thermoset materials," Polymer (Guildf)., vol. 153, pp. 653667, 2018, doi: 10.1016/j.polymer.2018.08.024.

[6] P. Musto, G. Ragosta, and G. Mensitieri, "Time-resolved FTIR/FTNIR spectroscopy: Powerful tools to investigate diffusion processes in polymeric films and membranes," e-Polymers, vol. 2, no. 1, 2002, doi: 10.1515/epoly.2002.2.1.220.

[7] M. Latino, F. Varela, Y. Tan, and M. Forsyth, "The effect of ageing on cathodic protection shielding by fusion bonded epoxy coatings," Prog. Org. Coatings, vol. 134, pp. 58-65, 2019.

[8] R. J. Varley and K. H. Leong, "Polymer Coatings for Oilfield Pipelines," in Active Protective Coatings: New-Generation Coatings for Metals, A. E. Hughes, J. M. C. Mol, M. L. Zheludkevich, and R. G. Buchheit, Eds. Dordrecht, Netherlands: Springer, 2016, pp. 385-428.

[9] J. A. Kehr, Fusion-bonded epoxy (FBE): a foundation for pipeline corrosion protection. Houston, Texas, USA: NACE International, 2003. 
[10] A. N. K. Jadoon and I. Thompson, "Fusion bonded epoxy mainline and field joint coatings performance from the X100 field trial - A case study," Int. J. Press. Vessel. Pip., vol. 92, pp. 4855, 2012, doi: 10.1016/j.jpvp.2012.01.003.

[11] G. R. Howell and Y. F. Cheng, "Characterization of high performance composite coating for the northern pipeline application," Prog. Org. Coatings, vol. 60, no. 2, pp. 148-152, 2007, doi: 10.1016/j.porgcoat.2007.07.013.

[12] T. Byrnes, "Pipeline coatings," in Trends in Oil and Gas Corrosion Research and Technologies, Elsevier Ltd., 2017, pp. 563-591.

[13] M. Latino, F. Varela, M. Forsyth, and Y. Tan, "Self-validating electrochemical methodology for quantifying ionic currents through pipeline coatings," Prog. Org. Coatings, vol. 120, pp. 153-159, 2018, doi: 10.1016/j.porgcoat.2018.03.018.

[14] M. Latino, F. Varela, Y. Tan, and M. Forsyth, "The effect of ageing on cathodic protection shielding by fusion bonded epoxy coatings," Prog. Org. Coatings, vol. 134, no. April, pp. 58-65, 2019, doi: 10.1016/j.porgcoat.2019.04.074.

[15] G. Bierwagen, D. Tallman, J. Li, L. He, and C. Jeffcoate, "EIS studies of coated metals in accelerated exposure," Prog. Org. Coatings, vol. 46, no. 2, pp. 149-158, 2003, doi: 10.1016/S0300-9440(02)00222-9.

[16] F. Mahdavi, M. Forsyth, and M. Y. J. Tan, "Techniques for testing and monitoring the cathodic disbondment of organic coatings: An overview of major obstacles and innovations," Prog. Org. Coatings, vol. 105, pp. 163-175, 2017, doi: 10.1016/j.porgcoat.2016.11.034.

[17] S. H. Lee, W. K. Oh, and J. G. Kim, "Acceleration and quantitative evaluation of degradation for corrosion protective coatings on buried pipeline: Part I. Development of electrochemical test methods," Prog. Org. Coatings, vol. 76, no. 4, pp. 778-783, 2013, doi: 10.1016/j.porgcoat.2012.06.010.

[18] M. Xu, C. N. C. Lam, D. Wong, and E. Asselin, "Evaluation of the cathodic disbondment resistance of pipeline coatings - A review," Prog. Org. Coatings, vol. 146, p. 105728, 2020, doi: 10.1016/j.porgcoat.2020.105728. 
[19] E. Legghe, E. Aragon, L. Bélec, A. Margaillan, and D. Melot, "Correlation between water diffusion and adhesion loss: Study of an epoxy primer on steel," Prog. Org. Coatings, vol. 66, no. 3, pp. 276-280, 2009, doi: 10.1177/1942602X15619756.

[20] C. N. C. Lam, D. T. Wong, R. E. Steele, and S. J. Edmondson, "A new approach to high performance polyolefin coatings," in Corrosion Conference and Expo, 2007, no. 07023.

[21] G. Z. Xiao, M. Delamar, and M. E. R. Shanahan, "Irreversible interactions between water and DGEBA / DDA epoxy resin during hygrothermal aging," J. Appl. Polym. Sci., vol. 65, no. 3, pp. 449-458, 1997.

[22] G. Z. Xiao and M. E. R. Shanahan, "Swelling of DGEBA/DDA epoxy resin during hygrothermal ageing," Polymer (Guildf)., vol. 39, no. 14, pp. 3253-3260, 1998, doi: 10.1016/S00323861(97)10060-X.

[23] C. Mo, W. Yuan, W. Lei, and Y. Shijiu, "Effects of temperature and humidity on the barrier properties of biaxially-oriented polypropylene and polyvinyl alcohol films," J. Appl. Packag. Res., vol. 6, no. 1, pp. 40-46, 2014, doi: 10.14448/japr.01.0004.

[24] Z. Zhang, I. J. Britt, and M. A. Tung, "Permeation of oxygen and water vapor through EVOH films as influenced by relative humidity," J. Appl. Polym. Sci., vol. 82, no. 8, pp. 1866-1872, 2001, doi: 10.1002/app.2030.

[25] S. Simone, A. Figoli, A. Criscuoli, M. C. Carnevale, A. Rosselli, and E. Drioli, "Preparation of hollow fibre membranes from PVDF/PVP blends and their application in VMD," J. Memb. Sci., vol. 364, no. 1-2, pp. 219-232, 2010, doi: 10.1016/j.memsci.2010.08.013.

[26] J. Lee, B. Park, J. Kim, and S. Bin Park, "Effect of PVP, lithium chloride, and glycerol additives on PVDF dual-layer hollow fiber membranes fabricated using simultaneous spinning of TIPS and NIPS," Macromol. Res., vol. 23, no. 3, pp. 291-299, 2015, doi: 10.1007/s13233-015-3037-x.

[27] O. Becker, R. J. Varley, and G. P. Simon, "Thermal stability and water uptake of high performance epoxy layered silicate nanocomposites," Eur. Polym. J., vol. 40, no. 1, pp. 187-195, 2004, doi: 10.1016/j.eurpolymj.2003.09.008.

[28] G. K. Van Der Wel and O. C. G. Adan, "Moisture in organic coatings - a review," Prog. Org. 
Coatings, vol. 37 , no. 1, pp. 1-14, 1999, doi: 10.1016/S0300-9440(99)00058-2.

[29] S. Sugiman and S. Salman, "Hygrothermal effects on tensile and fracture properties of epoxy filled with inorganic fillers having different reactivity to water," J. Adhes. Sci. Technol., vol. 33, no. 7, pp. 691-714, 2019, doi: 10.1080/01694243.2018.1558492.

[30] ASTM D1653-03, "Standard Test Methods for Water Vapor Transmission of Organic Coating Films," American Society for Testing Materials, West Conshohocken, PA, 2003.

[31] J. A. Kehr and D. G. Enos, "FBE, a foundation for pipeline corrosion coating," in Corrosion, 2000, pp. 1-20.

[32] D. R. Lide, CRC Handbook of Chemistry and Physics, 85th Edition, 85th ed. CRC Press, 2004.

[33] T. S. Chung, J. J. Shieh, W. W. Y. Lau, M. P. Srinivasan, and D. R. Paul, "Fabrication of multilayer composite hollow fiber membranes for gas separation," J. Memb. Sci., vol. 152, no. 2, pp. 211-225, 1999, doi: 10.1016/S0376-7388(98)00225-7.

[34] Z. Dai, L. Ansaloni, and L. Deng, "Recent advances in multi-layer composite polymeric membranes for CO2 separation: A review," Green Energy and Environment, vol. 1, no. 2. Elsevier Ltd, pp. 102-128, 2016, doi: 10.1016/j.gee.2016.08.001.

[35] A. Grüniger and P. R. Von Rohr, "Influence of defects in SiOx thin films on their barrier properties," Thin Solid Films, vol. 459, no. 1-2, pp. 308-312, 2004, doi: 10.1016/j.tsf.2003.12.146.

[36] R. W. Baker, J. G. Wijmans, and Y. Huang, "Permeability, permeance and selectivity: A preferred way of reporting pervaporation performance data," J. Memb. Sci., vol. 348, no. 1-2, pp. 346-352, 2010, doi: 10.1016/j.memsci.2009.11.022.

[37] F. A. Joy and A. G. Wilson, "Standardization of the dish method for measuring water vapor transmission," in International Symposium on Humidity and Moisture, Proceedings, 1963, pp. 259-270.

[38] H. N. Sharma, S. J. Harley, Y. Sun, and E. A. Glascoe, "Dynamic triple-mode sorption and outgassing in materials," Sci. Rep., vol. 7, no. 1, pp. 1-12, 2017, doi: 10.1038/s41598-01703091-3. 
[39] E. M. Davis and Y. A. Elabd, "Prediction of water solubility in glassy polymers using nonequilibrium thermodynamics," Ind. Eng. Chem. Res., vol. 52, pp. 12865-12875, 2013.

[40] S. Sato, M. Suzuki, S. Kanehashi, and K. Nagai, "Permeability, diffusivity, and solubility of benzene vapor and water vapor in high free volume silicon- or fluorine-containing polymer membranes," J. Memb. Sci., vol. 360, no. 1-2, pp. 352-362, 2010, doi: 10.1016/j.memsci.2010.05.029.

[41] C. A. Scholes, J. Jin, G. W. Stevens, and S. E. Kentish, "Competitive permeation of gas and water vapour in high free volume polymeric membranes," J. Polym. Sci. Part B Polym. Phys., vol. 53, no. 10 , pp. 719-728, 2015, doi: 10.1002/polb.23689.

[42] E. M. Davis and Y. A. Elabd, "Water clustering in glassy polymers," J. Phys. Chem. B, vol. 117, no. 36 , pp. 10629-10640, 2013, doi: 10.1021/jp405388d.

[43] G. Bouvet, N. Dang, S. Cohendoz, X. Feaugas, S. Mallarino, and S. Touzain, "Impact of polar groups concentration and free volume on water sorption in model epoxy free films and coatings," Prog. Org. Coatings, vol. 96, pp. 32-41, 2016, doi: 10.1016/j.porgcoat.2015.12.011.

[44] S. C. Chang and N. B. Hutcheon, "Dependence of water vapor permeability on temperature and humidity," Heating, Piping, Air Cond., no. 3, pp. 149-155, 1956.

[45] G. Q. Chen, C. A. Scholes, C. M. Doherty, A. J. Hill, G. G. Qiao, and S. E. Kentish, "Modeling of the sorption and transport properties of water vapor in polyimide membranes," J. Memb. Sci., vol. 409-410, pp. 96-104, 2012, doi: 10.1016/j.memsci.2012.03.047.

[46] A. Q. Fu and Y. F. Cheng, "Characterization of the permeability of a high performance composite coating to cathodic protection and its implications on pipeline integrity," Prog. Org. Coatings, vol. 72, no. 3, pp. 423-428, 2011, doi: 10.1016/j.porgcoat.2011.05.015.

[47] H. Zargarnezhad, E. Asselin, D. Wong, and C. Lam, "A critical review of the time-dependent performance of polymeric pipeline coatings: Focus on hydration of epoxy-based coatings," Polymers (Basel)., vol. 13, no. 9, p. 1517, 2021, doi: 10.3390/polym13091517.

[48] C. Carfagna and A. Apicella, "Physical degradation by water clustering in epoxy resins," J. Appl. Polym. Sci., vol. 28, pp. 2881-2885, 1983. 
[49] P. J. Flory, Principles of Polymer Chemistry. London: Cornell University Press, 1953.

[50] P. J. Flory, "Thermodynamics of high polymer solutions," J. Chem. Phys., vol. 9, no. 8, pp. 660661, 1941, doi: 10.1063/1.1750971.

[51] M. L. Huggins, "Solutions of long chain compounds," J. Chem. Phys., vol. 9, no. 5, p. 440, 1941, doi: $10.1063 / 1.1750930$.

[52] S. Maghami, A. Mehrabani-zeinabad, M. Sadeghi, and J. Sánchez-laínez, "Mathematical modeling of temperature and pressure effects on permeability, diffusivity and solubility in polymeric and mixed matrix membranes," Chem. Eng. Sci., vol. 205, pp. 58-73, 2019, doi: 10.1016/j.ces.2019.04.037.

[53] M. Minelli and G. C. Sarti, "Permeability and diffusivity of $\mathrm{CO} 2$ in glassy polymers with and without plasticization," J. Memb. Sci., vol. 435, pp. 176-185, 2013, doi: 10.1016/j.memsci.2013.02.013.

[54] A. F. Ismail and W. Lorna, "Penetrant-induced plasticization phenomenon in glassy polymers for gas separation membrane," Sep. Purif. Technol., vol. 27, pp. 173-194, 2002.

[55] A. Bos, I. G. M. Punt, M. Wessling, and H. Strathmann, "CO2-induced plasticization phenomena in glassy polymers," J. Memb. Sci., vol. 155, pp. 67-78, 1999.

[56] K. Oka, T. Shibue, N. Sugimura, Y. Watabe, B. Winther-Jensen, and H. Nishide, "Long-lived water clusters in hydrophobic solvents investigated by standard NMR techniques," Sci. Rep., vol. 9, no. 1, pp. 3-4, 2019, doi: 10.1038/s41598-018-36787-1.

[57] P. A. Saliba, A. A. Mansur, D. B. Santos, and H. S. Mansur, "Fusion-bonded epoxy composite coatings on chemically functionalized API steel surfaces for potential deep-water petroleum exploration," Appl. Adhes. Sci., vol. 3, no. 1, 2015, doi: 10.1186/s40563-015-0052-2.

[58] S. Sugiman, S. Salman, and M. Maryudi, "Effects of volume fraction on water uptake and tensile properties of epoxy filled with inorganic fillers having different reactivity to water," Mater. Today Commun., vol. 24, no. June, 2020, doi: 10.1016/j.mtcomm.2020.101360.

[59] A. Lekatou, S. E. Faidi, D. Ghidaoui, S. B. Lyon, and R. C. Newman, "Effect of water and its activity on transport properties of glass/epoxy particulate composites," Compos. Part A Appl. Sci. 
Manuf., vol. 28, no. 3, pp. 223-236, 1997, doi: 10.1016/S1359-835X(96)00113-3.

[60] C. Vosgien Lacombre, G. Bouvet, D. Trinh, X. Feaugas, S. Touzain, and S. Mallarino, "Influence of pigment and internal stresses on water uptake in model epoxy: a thermodynamic approach," $J$. Mater. Sci., vol. 53, no. 3, pp. 2253-2267, 2018, doi: 10.1007/s10853-017-1647-8.

[61] CSA Z245.20-18, "Plant-applied external fusion bond epoxy coating for steel pipe," 2018.

[62] A. Bahadori, "Chapter 8 - Materials and Construction for Three-Layer Polyethylene Coating Systems," in Essentials of Coating, Painting, and Lining for the Oil, Gas and Petrochemical Industries, Elsevier Inc., 2015, pp. 473-498.

[63] DIN 30670, "Polyethylene coatings on steel pipes and fittings - Requirements and testing," 2012.

[64] C. Yi, P. Rostron, N. Vahdati, E. Gunister, and A. Alfantazi, "Curing kinetics and mechanical properties of epoxy based coatings: The influence of added solvent," Prog. Org. Coatings, vol. 124, no. May, pp. 165-174, 2018, doi: 10.1016/j.porgcoat.2018.08.009.

[65] V. O. Startsev, M. P. Lebedev, K. A. Khrulev, M. V. Molokov, A. S. Frolov, and T. A. Nizina, "Effect of outdoor exposure on the moisture diffusion and mechanical properties of epoxy polymers," Polym. Test., vol. 65, no. November 2017, pp. 281-296, 2018, doi: 10.1016/j.polymertesting.2017.12.007.

[66] V. Sauvant-Moynot, J. Kittel, D. Melot, and M. Roche, "Three layer polyolefin coatings: How the FBE primer properties govern the long term adhesion," in 17th International Conference on Pipeline Protection, 2007, pp. 233-246.

[67] A. D. Drozdov, J. Christiansen, R. K. Gupta, and A. P. Shah, "Model for Anomalous Moisture Diffusion through a Polymer - Clay Nanocomposite," J. Polym. Sci. Part B Polym. Phys., vol. 41, pp. 476-492, 2003.

[68] D. F. Sanders et al., "Energy-efficient polymeric gas separation membranes for a sustainable future: A review," Polymer (Guildf)., vol. 54, no. 18, pp. 4729-4761, 2013, doi: 10.1016/j.polymer.2013.05.075.

[69] M. C. Celina and A. Quintana, "Oxygen diffusivity and permeation through polymers at elevated temperature," Polymer (Guildf)., vol. 150, pp. 326-342, 2018, doi: 
10.1016/j.polymer.2018.06.047.

[70] D. R. Paul, W. J. Koros, and C. Engineering, "Effect of partially immobilizing sorption on permeability and the diffusion time lag," J. Polym. Sci. Polym. Phys. Ed., vol. 14, pp. 675-685, 1976.

[71] N. Rajagopalan, C. E. Weinell, K. Dam-Johansen, and S. Kiil, "Influence of CO2at HPHT Conditions on the Properties and Failures of an Amine-Cured Epoxy Novolac Coating," Ind. Eng. Chem. Res., vol. 60, no. 41, pp. 14768-14778, 2021, doi: 10.1021/acs.iecr.1c02713.

[72] B. D. Freeman, "Basis of permeability/selectivity tradeoff relations in polymeric gas separation membranes," Macromolecules, vol. 32, no. 2, pp. 375-380, 1999, doi: 10.1021/ma9814548. 